\title{
Correction: Renzaho, A.M.N., et al. The Synergetic Effect of Targeted Resource Transfers for Families, Child Sensitive Social Protection Programs, and Capacity Building for Effective Social Protection on Children's Nutritional Status in Nepal. Int. J. Environ. Res. Public Health 2017, 14, 1502
}

\author{
Andre M. N. Renzaho ${ }^{1,2, *}$, Stanley Chitekwe ${ }^{3}$, Wen Chen ${ }^{1,4}$, Sanjay Rijal ${ }^{3}$, Thakur Dhakal ${ }^{3}$ \\ and Pradiumna Dahal ${ }^{3}$ \\ 1 Humanitarian and Development Studies, School of Social Sciences and Psychology, \\ Western Sydney University, Locked Bag 1797, Penrith NSW 2751, Australia; \\ Wen.Chen@westernsydney.edu.au \\ 2 School of Public Health and Preventive Medicine, Monash University, The Alfred Centre, \\ 99 Commercial Road, Melbourne VIC 3004, Australia \\ 3 UNICEF Nepal, Leknath Marg, Kathmandu 44600, Nepal; schitekwe@unicef.org (S.C.); \\ sarijal@unicef.org (S.R.); tdhakal@unicef.org (T.D.); pdahal@unicef.org (P.D.) \\ 4 Faculty of Medical Statistics and Epidemiology, School of Public Health, Sun Yat-sen University, \\ Guangzhou 510080, China \\ * Correspondence: Andre.Renzaho@westernsydney.edu.au
}

Received: 12 April 2018; Accepted: 15 April 2018; Published: 26 April 2018

The authors wish to add the following corrections to their paper published in the International Journal of Environmental Research and Public Health [1]. During the galley proof process, the production of the paper omitted the minus sign for the $95 \%$ CI of the results section on the project's impact on child underweight, wasting, and stunting in the abstract (p. 1) and the manuscript (p. 15).

In the abstract, the sentence regarding the result should be:

"Propensity score matched/weighted models produced better results than the unmatched analyses, and hence we report findings from the radius matching. The intervention resulted in a 5.2 (adjusted difference-in-difference [ADID] $=-5.16$; 95\% CI: $-9.55,-0.77$ ), 7.4 (ADID: -7.35 ; $95 \% \mathrm{CI}:-11.62,-3.08)$ and $2.8(\mathrm{ADID}=-2.84 ; 95 \% \mathrm{CI}:-5.58,-0.10)$ percentage point reduction in the prevalence of stunting, underweight, and wasting among children under the age of five, respectively. The intervention impact was greater in boys than girls for stunting and wasting; and greater in girls than boys for underweight. The intervention also resulted in a 6.7 (ADID $=-6.66 ; 95 \% \mathrm{CI}:-12.13$, -1.18), 11.4 (ADID $=-11.4 ; 95 \%$ CI: $-16.66,-6.13)$, and 4.1 (ADID $=-4.10 ; 95 \%$ CI: $-6.43,-1.78)$ percentage point reduction in the prevalence of stunting, underweight, and wasting among older children ( $\geq 24$ months). No impact was observed among younger children ( $<24$ months)."

The last two paragraphs in page 15 should be:

Our results suggest that the three matching estimators produced different effects on outcomes. The radius matching algorithm produced more robust results than the nearest neighbor or kernel matching estimators, and hence we report findings from the radius matching. The intervention had a positive impact on height-for-age z-scores (adjusted difference-in-difference (ADID) $=0.18 ; 95 \%$ CI: 0.09, 0.27, $p<0.05$ ), weight-for-age z-scores (ADID $=0.22,95 \% \mathrm{CI}: 0.15,0.19, p<0.01$ ), and weight-for-height z-scores (ADID $=0.19 ; 95 \%$ CI: 0.09, 0.30, $p<0.05)$. 
The intervention resulted in a $5.2(\mathrm{ADID}=-5.16 ; 95 \% \mathrm{CI}:-9.55,-0.77), 7.4$ (ADID: -7.35 ; 95\% CI: $-11.62,-3.08)$ and $2.8(\mathrm{ADID}=-2.84 ; 95 \% \mathrm{CI}:-5.58,-0.10)$ percentage point reduction in the proportion of children under the age of five who were stunted, underweight and wasted respectively. Among boys, the intervention resulted in a 6.2 (ADID = -6.15; 95\% CI: $-11.76,-0.53)$ and $3.3(\mathrm{ADID}=-3.33 ; 95 \% \mathrm{CI}:-6.16,-0.49)$ percentage point reduction in the prevalence of stunting and wasting respectively, but no impact was observed for underweight. Among girls, improvements were observed only for underweight, with a 9.0 (ADID $=-9.02 ; 95 \% \mathrm{CI}:-15.10,-2.94$ ) percentage point reduction in the prevalence of underweight. No impact was observed for stunting or wasting. The analysis by children's age groups revealed that the intervention resulted in a 6.7 (ADID = -6.66; 95\% CI: $-12.13,-1.18), 11.4$ (ADID = -11.40; 95\% CI: $-16.66,-6.13$ ), and 4.1 (ADID = -4.10; 95\% CI: $-6.43,-1.78)$ percentage point reduction in the prevalence of stunting, underweight, and wasting among older children ( $\geq 24$ months). No impact was observed among children younger than two years (Table 4; radius matching).

We deleted the word "baseline" in Figure 1:

\begin{tabular}{|c|c|c|}
\hline & \multicolumn{2}{|c|}{ Data analyses: Propensity score matching with difference-in-differences } \\
\hline & A & A \\
\hline Follow-up & $\begin{array}{l}\text { December 2014-February } 2015 \\
\checkmark \quad \text { Child anthropometric data, children } \\
<5 \text { years-weight and height } \\
\checkmark \quad \text { Demographic data: Child age and } \\
\text { gender, father and mother's } \\
\text { educational attainment and literacy } \\
\text { level, household wealth index, } \\
\text { caste/ethnicity, and expected food } \\
\text { shortage, and family size }\end{array}$ & $\begin{array}{l}\text { December 2014-February } 2015 \\
\checkmark \quad \text { Child anthropometric data, : children }<5 \\
\text { years-weight and height } \\
\checkmark \quad \text { Demographic data: Child age and } \\
\text { gender, father and mother's educational } \\
\text { attainment and literacy level, household } \\
\text { wealth index, caste/ethnicity, and } \\
\text { expected food shortage, and family size }\end{array}$ \\
\hline \multirow{3}{*}{ Baseline } & A & A \\
\hline & $\begin{array}{l}\text { October-December, } 2009 \\
\checkmark \quad \text { Child anthropometric data, children } \\
\quad<5 \text { years-weight and height } \\
\checkmark \quad \text { Demographic data: Child age and } \\
\text { gender, father and mother's } \\
\text { educational attainment and literacy } \\
\text { level, household wealth index, } \\
\text { caste/ethnicity, and expected food } \\
\text { shortage, and family size }\end{array}$ & $\begin{array}{l}\text { October-December, } 2009 \\
\checkmark \quad \text { Child anthropometric data, children }<5 \\
\text { years-weight and height } \\
\checkmark \quad \text { Demographic data: Child age and } \\
\text { gender, father and mother's educational } \\
\text { attainment and literacy level, household } \\
\text { wealth index, caste/ethnicity, and } \\
\text { expected food shortage, and family size }\end{array}$ \\
\hline & A & A \\
\hline Intervention & $\begin{array}{l}\text { Intervention received } \\
\text { Government's funded targeted resource } \\
\text { transfers (TRTs) for families: } \\
\text { - } \quad \text { Citizens allowance for all persons } \\
\text { - } \quad \text { Singed 70+ } \\
\text { - } \quad \text { Disability allowance for all people } \\
\text { with disability aged } 16 \text { years or older } \\
\text { - } \quad \text { Endangered ethnicities allowance } \\
\text { Maternity incentive scheme for } \\
\text { pregnant women and service } \\
\text { providers }\end{array}$ & 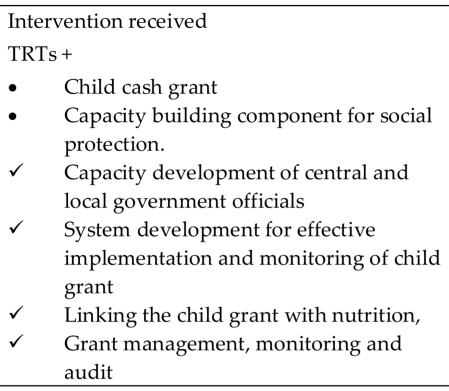 \\
\hline \multirow{4}{*}{ Enrolment/allocation } & A & A \\
\hline & \begin{tabular}{ll}
\multicolumn{2}{l}{ Bajhang District, Seti Zone } \\
- & Total population : 167,026 \\
- & Total number of households: 28,588 \\
- & Average family size: 5.8 \\
- & Tot literacy rate: $35.5 \%$ \\
- & $N=1500$ households \\
\end{tabular} & \begin{tabular}{ll}
\multicolumn{2}{l}{ Kalikot District, Karnali Zone; } \\
- & Total Population: 105,580 \\
- & Total number of households: 18,487 \\
- & Average family size: 5.7 \\
- & Total Literacy rate: $38.5 \%$ \\
- & $N=1500$ household \\
\end{tabular} \\
\hline & A & A \\
\hline & Comparison & Intervention \\
\hline
\end{tabular}

Figure 1. Flow diagram detailing the intervention implementation plan and data collection phases.

We also made some changes on Tables 2-4; therefore, the Tables should be as follows: 
Table 2. Summary statistics of the matching variables and estimates of logit regression models for stage 1 of propensity score matching.

\begin{tabular}{|c|c|c|c|c|c|c|c|c|c|c|c|c|c|c|c|c|c|c|}
\hline \multirow{3}{*}{ Matching Variables } & \multicolumn{3}{|c|}{ All } & \multicolumn{6}{|c|}{ Intervention } & \multicolumn{6}{|c|}{ Control } & \multicolumn{3}{|c|}{ Logit Model } \\
\hline & \multirow[b]{2}{*}{$N$} & \multirow[b]{2}{*}{ Mean } & \multirow[b]{2}{*}{ SD } & \multicolumn{3}{|c|}{ Baseline } & \multicolumn{3}{|c|}{ Follow-Up } & \multicolumn{3}{|c|}{ Baseline } & \multicolumn{3}{|c|}{ Follow-Up } & \multirow[b]{2}{*}{ Coefficient } & \multirow[b]{2}{*}{ SE } & \multirow[b]{2}{*}{$p$-Value } \\
\hline & & & & $N$ & Mean & SD & $N$ & Mean & SD & $N$ & Mean & SD & $N$ & Mean & SD & & & \\
\hline People per household & 3000 & & & 750 & & & 750 & & & 750 & & & 750 & & & & & \\
\hline 4 people or less & & $15.3 \%$ & $36.0 \%$ & & $13.5 \%$ & $34.2 \%$ & & $21.2 \%$ & $40.9 \%$ & & $15.3 \%$ & $36.1 \%$ & & $15.3 \%$ & $36.1 \%$ & 0.40 & 0.14 & 0.004 \\
\hline 5-8 people & & $63.8 \%$ & $48.1 \%$ & & $64.8 \%$ & $47.8 \%$ & & $65.2 \%$ & $47.7 \%$ & & $60.5 \%$ & $48.9 \%$ & & $60.5 \%$ & $48.9 \%$ & 0.16 & 0.10 & 0.111 \\
\hline 9 people or above & & $20.8 \%$ & $40.6 \%$ & & $21.7 \%$ & $41.3 \%$ & & $13.6 \%$ & $34.3 \%$ & & $24.1 \%$ & $42.8 \%$ & & $24.1 \%$ & $42.8 \%$ & Ref & & \\
\hline $\begin{array}{l}\text { Household wealth } \\
\text { index }\end{array}$ & 2899 & & & 724 & & & 710 & & & 731 & & & 731 & & & & & \\
\hline Poor & & $60.0 \%$ & $49.0 \%$ & & $89.1 \%$ & $31.2 \%$ & & $54.2 \%$ & $49.9 \%$ & & $10.1 \%$ & $30.2 \%$ & & $10.1 \%$ & $30.2 \%$ & 2.17 & 0.13 & 0.000 \\
\hline Middle class & & $20.0 \%$ & $40.0 \%$ & & $9.7 \%$ & $29.6 \%$ & & $35.9 \%$ & $48.0 \%$ & & $23.9 \%$ & $42.7 \%$ & & $23.9 \%$ & $42.7 \%$ & 2.08 & 0.15 & 0.000 \\
\hline Rich & & $20.0 \%$ & $40.0 \%$ & & $1.2 \%$ & $11.1 \%$ & & $9.9 \%$ & $29.8 \%$ & & $65.9 \%$ & $47.4 \%$ & & $65.9 \%$ & $47.4 \%$ & Reference & & \\
\hline Child's age in months & 3000 & 27.98 & 15.53 & 750 & 28.66 & 15.36 & 750 & 28.4 & 15.71 & 750 & 28.08 & 15.4 & 750 & 28.08 & 15.4 & 0.01 & 0.00 & 0.045 \\
\hline Child's gender & 3000 & & & 750 & & & 750 & & & 750 & & & 750 & & & & & \\
\hline Girl & & $43.4 \%$ & $49.6 \%$ & & $44.8 \%$ & $49.8 \%$ & & $43.6 \%$ & $49.6 \%$ & & $43.7 \%$ & $49.6 \%$ & & $43.7 \%$ & $49.6 \%$ & Reference & & \\
\hline Boy & & $56.6 \%$ & $49.6 \%$ & & $55.2 \%$ & $49.8 \%$ & & $56.4 \%$ & $49.6 \%$ & & $56.3 \%$ & $49.6 \%$ & & $56.3 \%$ & $49.6 \%$ & -0.08 & 0.08 & 0.322 \\
\hline Ethnicity & 3000 & & & 750 & & & 750 & & & 750 & & & 750 & & & & & \\
\hline $\begin{array}{c}\text { Disadvantage ethnic } \\
\text { groups }\end{array}$ & & $0.4 \%$ & $6.6 \%$ & & $1.5 \%$ & $12.0 \%$ & & $0.1 \%$ & $3.7 \%$ & & $0.0 \%$ & $0.0 \%$ & & $0.0 \%$ & $0.0 \%$ & 2.04 & 1.04 & 0.050 \\
\hline Dalit Hill/Terai & & $21.1 \%$ & $40.8 \%$ & & $21.3 \%$ & $41.0 \%$ & & $25.5 \%$ & $43.6 \%$ & & $16.8 \%$ & $37.4 \%$ & & $16.8 \%$ & $37.4 \%$ & 0.01 & 0.10 & 0.911 \\
\hline Upper caste Group & & $78.5 \%$ & $41.1 \%$ & & $77.2 \%$ & $42.0 \%$ & & $74.4 \%$ & $43.7 \%$ & & $83.2 \%$ & $37.4 \%$ & & $83.2 \%$ & $37.4 \%$ & Reference & & \\
\hline Father's education & 3000 & & & 750 & & & 750 & & & 750 & & & 750 & & & & & \\
\hline Intermediate or higher & & $12.6 \%$ & $33.2 \%$ & & $2.1 \%$ & $14.5 \%$ & & $16.8 \%$ & $37.4 \%$ & & $5.6 \%$ & $23.1 \%$ & & $25.9 \%$ & $43.8 \%$ & Reference & & \\
\hline Secondary level & & $30.0 \%$ & $45.8 \%$ & & $33.1 \%$ & $47.1 \%$ & & $22.3 \%$ & $41.6 \%$ & & $38.3 \%$ & $48.6 \%$ & & $26.4 \%$ & $44.1 \%$ & -0.05 & 0.14 & 0.744 \\
\hline Primary or less & & $57.4 \%$ & $49.5 \%$ & & $64.8 \%$ & $47.8 \%$ & & $60.9 \%$ & $48.8 \%$ & & $56.1 \%$ & $49.7 \%$ & & $47.7 \%$ & $50.0 \%$ & 0.27 & 0.14 & 0.052 \\
\hline
\end{tabular}


Table 3. Evaluation of standardized differences in matched sample.

\begin{tabular}{|c|c|c|c|c|c|}
\hline & \multicolumn{2}{|c|}{ Intervention } & \multicolumn{2}{|c|}{ Comparison } & \multirow{2}{*}{$\%$ Bias } \\
\hline & Unmatched & Matched & Unmatched & Matched & \\
\hline \multicolumn{6}{|l|}{ No. of people per household } \\
\hline 4 people or less & 0.159 & 0.082 & 0.128 & 0.118 & -10.30 \\
\hline 5-8 people & 0.657 & 0.664 & 0.629 & 0.659 & 1.20 \\
\hline 9 people or more & 0.184 & 0.254 & 0.243 & 0.223 & 7.40 \\
\hline \multicolumn{6}{|l|}{ Household wealth index } \\
\hline Poor & 0.717 & 0.648 & 0.484 & 0.683 & -7.40 \\
\hline Middle class & 0.227 & 0.275 & 0.174 & 0.240 & 8.80 \\
\hline Rich & 0.056 & 0.077 & 0.342 & 0.077 & 0.00 \\
\hline Child's age in months & 28.341 & 25.429 & 27.476 & 27.602 & -14.00 \\
\hline \multicolumn{6}{|l|}{ Child's gender } \\
\hline Girl & 0.438 & 0.395 & 0.429 & 0.421 & 5.30 \\
\hline Boy & 0.562 & 0.605 & 0.571 & 0.579 & -5.30 \\
\hline \multicolumn{6}{|l|}{ Ethnicity } \\
\hline Disadvantage ethnic groups & 0.008 & 0.001 & 0.001 & 0.001 & 0.00 \\
\hline Dalit Hill/Terai & 0.224 & 0.208 & 0.179 & 0.212 & -1.00 \\
\hline Upper caste Group & 0.768 & 0.791 & 0.820 & 0.787 & 1.00 \\
\hline \multicolumn{6}{|l|}{ Father's education } \\
\hline Primary or less & 0.630 & 0.496 & 0.519 & 0.540 & -8.90 \\
\hline Secondary level & 0.277 & 0.378 & 0.323 & 0.338 & 8.70 \\
\hline Intermediate or higher & 0.093 & 0.127 & 0.158 & 0.122 & -1.20 \\
\hline
\end{tabular}


Table 4. Program impact on child undernutrition.

\begin{tabular}{|c|c|c|c|c|c|c|c|c|c|c|c|c|c|c|c|c|}
\hline & \multicolumn{7}{|c|}{ Original Dataset } & \multicolumn{9}{|c|}{ Matched Dataset: Matching Algorithms } \\
\hline & \multirow{2}{*}{$\begin{array}{c}\text { Comparison } \\
N=748\end{array}$} & \multirow{2}{*}{$\begin{array}{c}\text { Intervention } \\
N=743 \\
\end{array}$} & \multirow{2}{*}{$\begin{array}{c}\text { Comparison } \\
N=749 \\
\end{array}$} & \multirow{2}{*}{$\begin{array}{c}\text { Intervention } \\
N=750\end{array}$} & \multirow[b]{2}{*}{ ADID } & \multirow[b]{2}{*}{$95 \% \mathrm{CI}$} & & \multicolumn{3}{|c|}{ Kernel! } & \multicolumn{3}{|c|}{ Nearest Neighbor! } & \multicolumn{2}{|c|}{ Radius !\# } & \\
\hline & & & & & & & & ADID & $95 \% \mathrm{CI}$ & & ADID & $95 \% \mathrm{CI}$ & & ADID & $95 \% \mathrm{CI}$ & \\
\hline \multicolumn{17}{|l|}{ Girls $^{\text {a }}$} \\
\hline Height & $77.2(10.3)$ & $77.8(10.9)$ & $78.7(11.1)$ & $78.8(11.7)$ & 0.17 & -0.05 & 0.40 & 0.65 & -0.87 & 2.18 & 0.01 & -1.43 & 1.45 & 0.69 & -0.99 & 2.36 \\
\hline Weight & $9.3(2.4)$ & $9.3(2.5)$ & $9.7(2.6)$ & $9.8(2.9)$ & $0.31 * * *$ & 0.22 & 0.40 & 0.32 & -0.06 & 0.71 & 0.13 & -0.25 & 0.51 & $0.33 *$ & 0.06 & 0.6 \\
\hline HAZ & $-2.3(1.3)$ & $-2.6(1.4)$ & $-2.1(1.3)$ & $-2.2(1.3)$ & 0.21 & -0.01 & 0.44 & 0.11 & -0.06 & 0.27 & 0.07 & -0.18 & 0.32 & 0.15 & -0.06 & 0.36 \\
\hline WAZ & $-1.7(1.0)$ & $-2.1(1.1)$ & $-1.5(1.1)$ & $-1.6(1.1)$ & $0.33^{* * *}$ & 0.23 & 0.44 & $0.17 *$ & 0.06 & 0.28 & 0.13 & -0.1 & 0.37 & $0.19 *$ & 0.09 & 0.29 \\
\hline WHZ & $-0.5(0.9)$ & $-0.8(1.1)$ & $-0.5(1.0)$ & $-0.4(1.0)$ & 0.31 *** & 0.15 & 0.46 & $0.17^{*}$ & 0.05 & 0.3 & 0.13 & -0.06 & 0.33 & 0.18 & -0.01 & 0.36 \\
\hline Stunting & 61.9 & 68 & 55.5 & 61 & -3.98 & -15.44 & 7.48 & -2.65 & -9.15 & 3.85 & -5.07 & -11.78 & 1.63 & -4.24 & -10.4 & 1.93 \\
\hline Underweight & 37.1 & 53.1 & 30.8 & 34.9 & $-16.25^{* * *}$ & -24.12 & -8.38 & $-7.83^{* * *}$ & -14.39 & -1.26 & -8.89 & -18.96 & 1.17 & $-9.02 * * *$ & -15.1 & -2.94 \\
\hline Wasting & 4.5 & 9.3 & 7 & 4.9 & $-9.29 * * *$ & -15.86 & -2.72 & -2.62 & -6.33 & 1.09 & -3.31 & -8.2 & 1.58 & -2.47 & -5.9 & 0.95 \\
\hline \multicolumn{17}{|l|}{ Boys ${ }^{a}$} \\
\hline Height & $80.2(11.2)$ & $80.6(11.2)$ & 82.4 (11.2) & $81.6(11.8)$ & -0.05 & -1.17 & 1.06 & 0.21 & -1.31 & 1.74 & 0.13 & -1.13 & 1.39 & 0.22 & -0.9 & 1.35 \\
\hline Weight & $10.2(2.6)$ & $10.2(2.7)$ & $10.9(2.8)$ & $10.7(3.0)$ & 0.17 & -0.17 & 0.52 & 0.23 & -0.11 & 0.57 & 0.21 & -0.23 & 0.66 & 0.25 & -0.09 & 0.6 \\
\hline HAZ & $-2.4(1.3)$ & $-2.6(1.5)$ & $-2.0(1.3)$ & $-2.2(1.4)$ & 0.14 & -0.14 & 0.43 & $0.16^{*}$ & 0 & 0.31 & 0.08 & -0.17 & 0.33 & 0.22 * & 0.08 & 0.35 \\
\hline WAZ & $-1.7(1.0)$ & $-2.1(1.1)$ & $-1.4(1.1)$ & $-1.6(1.1)$ & 0.26 & 0.01 & 0.51 & $0.19 * *$ & 0.1 & 0.29 & $0.17^{*}$ & 0.01 & 0.32 & $0.25 *$ & 0.08 & 0.42 \\
\hline WHZ & $-0.6(0.9)$ & $-0.9(1.2)$ & $-0.3(1.1)$ & $-0.4(1.0)$ & $0.27 * * *$ & 0.08 & 0.47 & 0.21 * & 0.06 & 0.36 & 0.20 * & 0.02 & 0.38 & 0.21 * & 0.07 & 0.36 \\
\hline Stunting & 63.7 & 65.7 & 50.8 & 58.8 & 0.69 & -14.00 & 15.37 & -4.14 & -10.48 & 2.19 & -1.27 & -10.49 & 7.95 & $-6.15^{*}$ & -11.76 & -0.53 \\
\hline Underweight & 37.4 & 48.8 & 27.5 & 34.8 & -9.74 & -23.38 & 3.90 & -5.03 & -11.19 & 1.13 & -3.39 & -13.45 & 6.67 & -6.49 & -13.15 & 0.16 \\
\hline Wasting & 6.6 & $15.3^{* * * *}$ & 5.9 & 6.4 & $-9.55 * * *$ & -14.46 & -4.64 & -3.11 & -6.4 & 0.19 & -3.54 & -8.31 & 1.23 & $-3.33^{*}$ & -6.16 & -0.49 \\
\hline \multicolumn{17}{|l|}{$<2$ years $^{b}$} \\
\hline Height & $70.0(6.5)$ & $69.6(6.7)$ & $70.8(7.4)$ & $69.2(7.4)$ & -0.28 & -1.16 & 0.60 & $-0.85^{*}$ & -1.67 & -0.02 & -0.91 & -2.45 & 0.63 & -0.81 * & -1.6 & -0.02 \\
\hline Weight & $7.8(1.5)$ & 7.5 (1.6) & $8.1(1.8)$ & $7.6(1.8)$ & 0.03 & -0.30 & 0.37 & -0.15 & -0.38 & 0.08 & -0.17 & -0.45 & 0.11 & -0.14 & -0.36 & 0.08 \\
\hline HAZ & $-2.0(1.4)$ & $-2.2(1.5)$ & $-1.6(1.4)$ & $-1.9(1.5)$ & 0.03 & -0.21 & 0.28 & 0.12 & -0.09 & 0.33 & -0.1 & -0.37 & 0.18 & 0.13 & -0.08 & 0.33 \\
\hline WAZ & $-1.5(1.1)$ & $-2.0(1.2)$ & $-1.2(1.2)$ & $-1.6(1.2)$ & 0.18 & -0.04 & 0.41 & 0.08 & -0.06 & 0.22 & -0.01 & -0.24 & 0.23 & 0.09 & -0.08 & 0.27 \\
\hline WHZ & $-0.6(0.9)$ & $-1.1(1.3)$ & $-0.5(1.1)$ & $-0.7(1.1)$ & 0.18 & -0.04 & 0.41 & 0.05 & -0.09 & 0.2 & 0.1 & -0.15 & 0.34 & 0.07 & -0.08 & 0.21 \\
\hline Stunting & 52 & 58.2 & 39.8 & 50.8 & 2.76 & -5.16 & 10.68 & -2.48 & -8.1 & 3.14 & 1.61 & -6.44 & 9.66 & -3.57 & -10.37 & 3.23 \\
\hline Underweight & 32.6 & 47.1 & 23.8 & 37.1 & -5.39 & -18.43 & 7.66 & -0.46 & -7.8 & 6.89 & 1.86 & -8.42 & 12.15 & -1.24 & -8.08 & 5.6 \\
\hline Wasting & 6.7 & 18.8 & 6.8 & 10.3 & $-9.19 * * *$ & -15.81 & -2.57 & -1.2 & -5.16 & 2.76 & -1.91 & -6.88 & 3.05 & -1.03 & -4.2 & 2.13 \\
\hline \multicolumn{17}{|l|}{$\geq 2$ years ${ }^{b}$} \\
\hline Height & $87.1(7.1)$ & $86.4(7.9)$ & $88.3(7.1)$ & $87.9(7.6)$ & 0.53 & -0.12 & 1.18 & 0.41 & -0.18 & 1.01 & 0.59 & -0.45 & 1.63 & 0.74 & -0.16 & 1.64 \\
\hline Weight & $11.7(1.9)$ & $11.4(2.0)$ & $12.1(2.0)$ & $12.1(2.1)$ & $0.39 * * *$ & 0.12 & 0.66 & $0.36 * * *$ & 0.12 & 0.6 & $0.44^{* *}$ & 0.18 & 0.69 & $0.44 * * *$ & 0.25 & 0.63 \\
\hline HAZ & $-2.6(1.1)$ & $-2.8(1.2)$ & $-2.4(1.1)$ & $-2.4(1.3)$ & 0.15 & -0.02 & 0.31 & $0.17 *$ & 0.06 & 0.28 & 0.12 & -0.03 & 0.28 & 0.21 * & 0.06 & 0.35 \\
\hline WAZ & $-1.9(1.0)$ & $-2.1(1.1)$ & $-1.6(1.0)$ & $-1.6(1.0)$ & $0.28 * * *$ & 0.12 & 0.44 & $0.28^{* * *}$ & 0.18 & 0.37 & $0.27 * *$ & 0.13 & 0.41 & $0.30^{* * *}$ & 0.19 & 0.41 \\
\hline WHZ & $-0.5(0.9)$ & $-0.6(1.0)$ & $-0.3(1.0)$ & $-0.2(0.9)$ & 0.29 *** & 0.11 & 0.47 & $0.26^{* * *}$ & 0.17 & 0.35 & $0.29 * *$ & 0.12 & 0.46 & $0.27 * * *$ & 0.14 & 0.4 \\
\hline Stunting & 73 & 73.1 & 62.8 & 65.8 & 0.05 & -6.01 & 6.11 & -4.82 & -10.23 & 0.6 & -4.05 & -12.54 & 4.44 & $-6.66^{* *}$ & -12.13 & -1.18 \\
\hline Underweight & 41.5 & 53.3 & 32.8 & 33.3 & $-14.87^{* * *}$ & -23.27 & -6.46 & $-10.45^{* * *}$ & -16.02 & -4.88 & -9.2 & -18.52 & 0.11 & $-11.40^{* * *}$ & -16.66 & -6.13 \\
\hline Wasting & 4.9 & 8.2 & 6.1 & 2.7 & $-8.51 * * *$ & -13.91 & -3.11 & $-3.86^{* *}$ & -5.98 & -1.74 & $-6.22 * *$ & -9.22 & -3.22 & $-4.10 * *$ & -6.43 & -1.78 \\
\hline
\end{tabular}


Table 4. Cont.

\begin{tabular}{|c|c|c|c|c|c|c|c|c|c|c|c|c|c|c|c|c|}
\hline $\mathrm{All}^{\mathrm{c}}$ & & & & & & & & & & & & & & & & \\
\hline Height & 78.9 (10.9) & $79.3(11.1)$ & $80.8(11.3)$ & $80.3(11.9)$ & 0.11 & -0.51 & 0.72 & 0.42 & -0.68 & 1.52 & -0.11 & -1.08 & 0.86 & 0.48 & -0.33 & 1.28 \\
\hline Weight & $9.8(2.6)$ & $9.8(2.7)$ & $10.4(2.8)$ & $10.3(3.0)$ & $0.26 * *$ & 0.05 & 0.47 & $0.27 *$ & 0 & 0.55 & 0.17 & -0.12 & 0.47 & 0.29 & -0.01 & 0.6 \\
\hline HAZ & $-2.3(1.3)$ & $-2.6(1.4)$ & $-2.1(1.3)$ & $-2.2(1.4)$ & 0.17 * & 0.03 & 0.31 & 0.14 * & 0.03 & 0.25 & 0.05 & -0.12 & 0.23 & 0.18 * & 0.09 & 0.27 \\
\hline WAZ & $-1.7(1.0)$ & $-2.1(1.1)$ & $-1.4(1.1)$ & $-1.6(1.1)$ & $0.29^{* * * *}$ & 0.15 & 0.44 & $0.19 * *$ & 0.11 & 0.28 & $0.18^{*}$ & 0.07 & 0.29 & 0.22 ** & 0.15 & 0.29 \\
\hline WHZ & $-0.5(0.9)$ & $-0.8(1.1)$ & $\begin{array}{l}-1.4(1.1) \\
-0.4(1.1)\end{array}$ & $-0.4(1.0)$ & $0.29 * * *$ & 0.15 & $\begin{array}{l}0.44 \\
0.42\end{array}$ & $0.18 *$ & $\begin{array}{l}0.11 \\
0.09\end{array}$ & 0.28 & 0.24 * & 0.08 & 0.4 & $0.19 *$ & 0.09 & 0.3 \\
\hline Stunting & 63 & 66.7 & 52.9 & 59.8 & -1.34 & -7.12 & 4.44 & -3.51 & -7.83 & 0.82 & -2.18 & -10.22 & 5.87 & $-5.16^{*}$ & -9.55 & -0.77 \\
\hline Underweight & 37.3 & 50.7 & 28.9 & 34.8 & $-12.54 * * *$ & -19.82 & -5.25 & $-6.29 * * *$ & -10.96 & -1.62 & -5.19 & -10.75 & 0.37 & $-7.35 * * *$ & -11.62 & -3.08 \\
\hline Wasting & 5.8 & 12.7 & 6.4 & 5.7 & $-9.32^{* * * *}$ & -14.86 & -3.79 & $-2.86^{*}$ & -4.91 & -0.8 & $-4.84 * * *$ & -8.62 & -1.06 & -2.84 ** & -5.58 & -0.1 \\
\hline
\end{tabular}
family size; weighted with bootstrapping; ${ }^{b}$ Adjusted for father's educational attainment, household wealth index, caste/ethnicity, gender, and family size, weighted with bootstrapping; c Adjusted for father's educational attainment, household wealth index, caste/ethnicity, gender, child age in month, and family size, weighted with bootstrapping. \# Radius = 0.02; ! Weighted with bootstrapping. Z scores for height-for-age (HAZ), weight-for-age (WAZ) and weight-height (WHZ). 
We apologize for any inconvenience caused to the readers by this error.

Author Contributions: This study was designed and implemented by UNICEF Nepal. A.M.N.R. carried out the analyses and drafted the manuscript. All authors critically revised the manuscript for intellectual contents, and read and approved the final manuscript.

Conflicts of Interest: The authors declare no conflict of interest.

\section{Reference}

1. Renzaho, A.M.N.; Chitekwe, S.; Chen, W.; Rijal, S.; Dhakal, T.; Dahal, P. The Synergetic Effect of Cash Transfers for Families, Child Sensitive Social Protection Programs, and Capacity Building for Effective Social Protection on Children's Nutritional Status in Nepal. Int. J. Environ. Res. Public Health 2017, 14, 1502. [CrossRef] [PubMed]

(C) 2018 by the authors. Licensee MDPI, Basel, Switzerland. This article is an open access article distributed under the terms and conditions of the Creative Commons Attribution (CC BY) license (http:/ / creativecommons.org/licenses/by/4.0/). 